\title{
Robust second-order rotatable designs invariably applicable for some lifetime distributions
}

\author{
Jinseog $\operatorname{Kim}^{a}$, Rabindra Nath Das ${ }^{1, b}$, Poonam Singh ${ }^{c}$, Youngjo Lee ${ }^{d}$ \\ ${ }^{a}$ Department of Big data and Applied Statistics, Dongguk University, Korea; \\ ${ }^{b}$ Department of Statistics, The University of Burdwan, India; \\ ${ }^{c}$ Department of Statistics, University of Delhi, India; \\ ${ }^{d}$ Seoul National University and Dankook University, Korea
}

\begin{abstract}
Recently a few articles have derived robust first-order rotatable and D-optimal designs for the lifetime response having distributions gamma, lognormal, Weibull, exponential assuming errors that are correlated with different correlation structures such as autocorrelated, intra-class, inter-class, tri-diagonal, compound symmetry. Practically, a first-order model is an adequate approximation to the true surface in a small region of the explanatory variables. A second-order model is always appropriate for an unknown region, or if there is any curvature in the system. The current article aims to extend the ideas of these articles for second-order models. Invariant (free of the above four distributions) robust (free of correlation parameter values) second-order rotatable designs have been derived for the intra-class and inter-class correlated error structures. Second-order rotatability conditions have been derived herein assuming the response follows non-normal distribution (any one of the above four distributions) and errors have a general correlated error structure. These conditions are further simplified under intra-class and inter-class correlated error structures, and second-order rotatable designs are developed under these two structures for the response having anyone of the above four distributions. It is derived herein that robust second-order rotatable designs depend on the respective error variance covariance structure but they are independent of the correlation parameter values, as well as the considered four response lifetime distributions.
\end{abstract}

Keywords: correlated errors, invariant designs, lifetime distributions, mean lifetime, response surface, robust second-order rotatability

\section{Introduction}

Generally, response surface methodology (RSM) assumes that the response follows normal distribution with independent and homoscedastic errors (Box and Hunter 1957; Box and Draper 2007). RSM is frequently used in lifetime improvement (or quality engineering) experiments for locating the optimal level combinations to attain the specific target (Nair et al., 1992; Myers et al., 2002), while the response lifetime generally follows exponential, lognormal Weibull, gamma distributions (Lawless, 1982; Das and Lee 2009; Das 2013). In addition, lifetime observations can be correlated (Myers et al., 2002). Thus, usual RSM is not appropriate for lifetime improvement experiments. Second-order rotatable designs with correlated errors, assuming a response that follows normal distribution was introduced by Das (1997). First-order D-optimal and rotatable designs with correlated errors for some lifetime distributions have been studied by Das and Lin (2011), Das and Huda (2011), Das, Kim and

\footnotetext{
${ }^{1}$ Corresponding author: Department of Statistics, The University of Burdwan, Rajbati, Bardhaman, West Bengal 713104, India. E-mail: rabin.bwn@gmail.com
}

Published 30 November 2021 / journal homepage: http://csam.or.kr

(C) 2021 The Korean Statistical Society, and Korean International Statistical Society. All rights reserved. 
Park (2015), and Das, Kim and Lee (2015). Note that second-order D-optimal slope-rotatable designs under normal distributions have been studied by Das et al., (2010). First-order models are not appropriate for unknown experimental regions, whereas second-order models are always suitable. The present article considers a correlated second-order lifetime model having the above mentioned four lifetime distributions, and errors having intra and inter-class structures. Invariant robust second-order rotatable designs (RSORDs) have been derived for these situations.

The article considers four lifetime distributions such as lognormal, gamma, exponential, Weibull and to be added. Suppose the lifetime $\left(T_{1}\right)$ follows a Weibull distribution with the probability density function (p.d.f.):

$$
\frac{\delta_{1}}{\alpha_{1}}\left(\frac{t_{1}}{\alpha_{1}}\right)^{\delta_{1}-1} \exp \left[-\left(\frac{t_{1}}{\alpha_{1}}\right)^{\delta_{1}}\right], \quad t_{1} \geq 0 .
$$

Suppose $k$ concomitant control experimental factors (or variables) noted as $\mathbf{z}=\left(z_{1}, z_{2}, \ldots, z_{k}\right)^{\prime}$ are adopted to explain the heterogeneity in lifetime $T_{1}$. Commonly used Weibull regression models are those for which just $\alpha_{1}$, but not $\delta_{1}$, depends on $\mathbf{z}$, and $\alpha_{1}$ can be replaced by $\alpha_{1}(\mathbf{z})$. Therefore, the p.d.f. of $X_{0}=\ln T_{1}$, given $\mathbf{z}$ is as follows,

$$
f\left(x_{0} \mid \mathbf{z}\right)=\frac{1}{\sigma} \exp \left[\frac{x_{o}-\mu(\mathbf{z})}{\sigma}-\exp \left(\frac{x_{0}-\mu(\mathbf{z})}{\sigma}\right)\right], \quad-\infty<x_{0}<\infty,
$$

where $\sigma=1 / \delta_{1}$ and $\mu(\mathbf{z})=\ln \alpha_{1}(\mathbf{z})$. From the above distribution of $X_{0} \mid \mathbf{z}$, it can be written as

$$
X_{0}=\mu(\mathbf{z})+\sigma h_{1},
$$

where $h_{1}$ follows the standard extreme value distribution with p.d.f.: $\exp \left(h_{1}-e^{h_{1}}\right),-\infty<h_{1}<\infty$. Note that $\mathrm{E}\left(h_{1}\right)=-v$, where $v=0.5772, \ldots$, known as Euler's constant and $\operatorname{Var}\left(h_{1}\right)=\pi^{2} / 6$. The model (1.1) is known as a location-scale regression model with random component $h_{1}$. A class of models can be derived for different choices of $\mu(\mathbf{z})=\ln \alpha_{1}(\mathbf{z})$.

If $\delta_{1}=1(\sigma=1)$, the above Weibull distribution reduces to exponential lifetime distribution, and (1.1) reduces to,

$$
X_{0}=\mu(\mathbf{z})+h_{1} .
$$

For gamma lifetime $\left(T_{1}\right)$ distribution with p.d.f.: $1 / \Gamma(p) t_{1}^{p-1} /\left\{\alpha_{1}(\mathbf{z})\right\}^{p} \exp \left[-t_{1} / \alpha_{1}(\mathbf{z})\right], \quad t_{1} \geq 0$, the p.d.f. of $X_{0}=\ln T_{1}$, given $\mathbf{z}$, is given by,

$$
f\left(x_{0} \mid \mathbf{z}\right)=\frac{1}{\Gamma(p)} \exp \left[\left(x_{0}-\mu(\mathbf{z})\right) p\right] \exp \left[-\exp \left(x_{0}-\mu(\mathbf{z})\right)\right], \quad-\infty<x_{0}<\infty,
$$

where $\Gamma(p)$ is a gamma function of $p$. Similarly, from the above distribution, it can be expressed as

$$
X_{0}=\mu(\mathbf{z})+h_{2},
$$

where $h_{2}$ follows log-gamma distribution with p.d.f.: $1 / \Gamma(p) \exp \left(h_{2} p-e^{h_{2}}\right),-\infty<h_{2}<\infty$.

For the lifetime $\left(T_{1}\right)$ follows lognormal distribution $\left(\ln T_{1}\left(=X_{0}\right)\right.$ follows normal distribution with mean $\ln \alpha_{1}(\mathbf{z})$ and variance $\delta_{2}^{2}$ ) with p.d.f.: $1 / t_{1} \delta_{2}(2 \pi)^{1 / 2} \exp \left[-\left(\ln t_{1}-\ln \alpha_{1}(\mathbf{z})\right)^{2} / 2 \delta_{2}^{2}\right], t_{1} \geq 0, \delta_{2} \geq$ 0 , then

$$
X_{0}=\mu(\mathbf{z})+\delta_{2} \tau,
$$


Table 1: Results for mean models of threading machine lifetime data from log-normal and gamma fits

\begin{tabular}{|c|c|c|c|c|c|c|c|c|c|}
\hline & \multirow[b]{2}{*}{ Covar. } & \multicolumn{4}{|c|}{ Log-normal model } & \multicolumn{4}{|c|}{ Gamma model } \\
\hline & & est. & s.e. & $t$ & $P$-value & est. & s.e. & $t$ & $P$-value \\
\hline Mean & const. & 4.10 & 0.11 & 37.46 & $<0.01$ & 4.37 & 0.11 & 39.73 & $<0.01$ \\
\hline \multirow{8}{*}{ Model } & $A$ & -0.84 & 0.11 & -7.66 & $<0.01$ & -0.84 & 0.11 & -7.55 & $<0.01$ \\
\hline & $B$ & 0.48 & 0.11 & 4.32 & $<0.01$ & 0.47 & 0.11 & 4.24 & $<0.01$ \\
\hline & C & 0.44 & 0.11 & 4.01 & $<0.01$ & 0.42 & 0.11 & 3.83 & $<0.01$ \\
\hline & $D$ & -0.18 & 0.11 & -1.66 & 0.10 & -0.14 & 0.11 & -1.28 & 0.21 \\
\hline & E & 0.04 & 0.11 & 0.38 & 0.71 & 0.07 & 0.11 & 0.59 & 0.55 \\
\hline & $C E$ & -0.36 & 0.11 & -3.30 & $<0.01$ & -0.31 & 0.11 & -2.76 & 0.01 \\
\hline & $D E$ & 0.22 & 0.11 & 1.96 & 0.05 & 0.18 & 0.11 & 1.65 & 0.10 \\
\hline & $A B$ & -0.19 & 0.11 & -1.71 & 0.09 & -0.17 & 0.11 & -1.58 & 0.12 \\
\hline $\begin{array}{c}\text { Var } \\
\text { model }\end{array}$ & const. & -0.44 & 0.20 & -2.19 & 0.03 & -0.54 & 0.20 & -2.64 & 0.01 \\
\hline AIC & & \multicolumn{4}{|c|}{599.0} & \multicolumn{4}{|c|}{604.4} \\
\hline
\end{tabular}

where $\tau$ follows the standard normal distribution.

In the following section, it is shown that for a real lifetime data set, the lifetime distribution is important for locating the optimal level combinations from its fitted second-order model with constant variance. The article is organized as follows. The following section presents a motivating example, while Sections 3 and 4 present correlated second-order model and rotatability conditions, respectively. Section 5 presents robust second-order rotatable designs, which is then followed by the conclusion.

\section{Motivating second-order real lifetime model example}

Firth (1988) has shown that the regression parameter estimates from the log-normal, or the gamma model have almost identical results with constant variance. In this section, a real lifetime experimental data set is presented which has different regression parameter estimates for these two models with constant variance. For non-constant variance, regression parameter estimates for these two models may be different which has been shown by Das and Lee (2009).

Example: Watkins et al. (1994) studied the lifetime of a cutting tool which was used to minimize the tooling costs of deducting threads on pipes to make electrical conduit. The cutting tool is known as a chaser and the angle at which it accesses the pipe is known as the rake angle. The experimental operating factors are chaser type $(A)$, coolant type $(B)$, speed $(C)$, conduit manufacturer $(D)$, and rake angle $(E)$. For each factor, two levels were used using a $2^{5-1}$ design with $E=A B C D$. The threading machine had two spindles (right and left denoted by $\mathrm{R}$ and $\mathrm{L}$ ); each spindle can handle a pipe. The study variable is the time until poor thread quality is observed or the product has to be scrapped. The lifetime data and design matrix are displayed in Watkins et al. (1994). It is noted that no observation was censored, and the setting number 4 had three runs, while the setting number 15 had two runs.

The lognormal and gamma model analyses of the above data set have been derived by Das and Park (2012), where the estimates of the two models are not similar, and also the model selection criteria (based on Akaike information criterion (AIC)) are not same. For reference, both analyses results are reproduced in Table 1. From Table 1, it is clear that the regression parameter estimates are not the same in both the models. In addition, the lognormal model gives a better fit than the gamma based on AIC. It is clear that $D E(5 \%)$ and $A B(9 \%)$ are almost significant in the lognormal model, while they are not significant in the gamma model. It has been shown that many second-order significant terms are present in the lognormal mean model, while the variance is constant. Thus, for a lifetime analysis, an appropriate second-order model is important for locating the optimal level combinations 
along with its distribution. Again some runs are replicated that may introduce correlation in the response lifetime data, which was pointed out by Myers et al. (2002). For these situations, appropriate second-order designs are very important in order to obtain better information regarding the regression parameters. To the best of our knowledge these problems (second-order correlated lifetime models and the respective designs) are little studied in the quality engineering literature. In the following sections, these problems are addressed.

\section{Correlated lifetime second-order models}

First-order models are often used if the experimenters are remote from the optimum operating conditions of the process. The direction of steepest ascent indicates the direction in which the estimated response $(\hat{x})$ increases most rapidly, under the assumption that response $x$ is a larger-the-better characteristic. If there is a curvature in the process, then a higher degree polynomial such as the secondorder model must be used. Each of the above location-scale model (equations (1.1) to (1.4)) has two components: $\mu(\mathbf{z})$ (systematic part) and a random component (connected to the original lifetime $\left(T_{1}\right)$ distribution). Note that the random component (in equations (1.1) to (1.4)) distribution is completely different from the original lifetime distribution of $T_{1}$, and it is not a usual 'random effect' (generally used in random effects model) such as unobserved effect, missing information, censored data, unknown future prediction etc. (Lee et al., 2017). Correlated second-order linear natural logarithm lifetime models under Weibull, exponential, lognormal and gamma distributions are derived as follows.

For a lifetime $T_{1}$ which follows Weibull distribution as in (1.1) with mean $\alpha_{1}(\mathbf{z})\left(=e^{g(\mathbf{z}, \boldsymbol{\beta})}\right.$, say as $t_{1}>0$ ) indicating $\mu(\mathbf{z})=\ln \alpha_{1}(\mathbf{z})=g(\mathbf{z}, \boldsymbol{\beta})$. This is the same as $X_{0}=\ln T_{1} \mid \mathbf{z}=g(\mathbf{z}, \boldsymbol{\beta})+\sigma h_{1}$ (from (1.1)), where $h_{1}$ follows the standard extreme value distribution. Assuming that the response surface $g(\mathbf{z}, \boldsymbol{\beta})$ is second-order, we adopt the model,

$$
\ln T_{1} \mid \mathbf{z}=\beta_{0}+\sum_{i=1}^{k} \beta_{i} z_{i}+\sum_{i \leq j}^{k} \beta_{i j} z_{i} z_{j}+\sigma h_{1},
$$

where $h_{1}$ follows the standard extreme value distribution whose mean and variance are given in (1.1). For estimating the parameters $\beta_{0}, \beta_{1}, \ldots, \beta_{k}, \beta_{11}, \ldots, \beta_{k k}, \beta_{12}, \ldots, \beta_{(k-1) k}$ one needs to conduct an experiment to collect the data on the basis of which the estimation is to be done. As soon as an experiment is conducted, the experiment provides some noise factors which may be numerous, some of which may be even unidentifiable or undefinable. The whole effect of all these noise factors on the response ' $\ln T_{1}$ ' expressed by the experimental condition is denoted by ' $e$ ' and is known as an experimental error. It is written as,

$$
\begin{gathered}
y=\ln T_{1} \mid \mathbf{z}+e=\beta_{0}+\sum_{i=1}^{k} \beta_{i} z_{i}+\sum_{i \leq j}^{k} \beta_{i j} z_{i} z_{j}+\sigma h_{1}+e, \\
\text { or , } y_{u}=\beta_{0}+\sum_{i=1}^{k} \beta_{i} z_{u i}+\sum_{i \leq j}^{k} \beta_{i j} z_{u i} z_{u j}+\sigma h_{1 u}+e_{u}, \quad 1 \leq u \leq N,
\end{gathered}
$$

where $\beta_{i}$ are unknown regression parameters and $z_{u i}$ (for the $u^{\text {th }}$ run, $1 \leq u \leq N$, of $i^{\text {th }}$ factor, $1 \leq i \leq k$ ) are experimental levels which are non-stochastic, $h_{1}$ follows standard extreme value distribution, and $e_{u}$ 's represent experimental errors that arise from the noise factors. Note that there are two random 
variables ' $h_{1}$ ' and ' $e$ ' in the model (3.1), where ' $h_{1}$ ' is associated with the original lifetime $T_{1}$ distribution, and ' $\mathrm{e}$ ' is the random error which accounts for all sources of variation due to noise factors included in the experiment. The model (3.1) is a linear mixed model. Note that not one of these two random variables is the same as random effects generally used in random effect models such as generalized linear mixed models, hierarchical generalized linear models (HGLMs) or double HGLMs (Lee et al., 2017). Usual second-order response surface models do not consider the random component related to the lifetime distribution. It is assumed that $h_{1 u}$ 's are all uncorrelated and $h_{1 u}$ and $e_{u}$ 's are uncorrelated. Following usual response surface models (Box and Hunter 1957), it is assumed that $e_{u}$ 's follows normal distribution. In addition, this article assumes that $e_{u}$ 's are not independent but they are correlated, which is frequently observed in practice (Myers et al., 2002). Therefore, $\mathbf{e}=\left(e_{1}, \ldots, e_{N}\right)^{T}$ follows the multivariate normal distribution with $E(\mathbf{e})=\mathbf{0}$, dispersion (variance-covariance) matrix, $\operatorname{Dis}(\mathbf{e})=\sigma_{1}^{2} W_{1}$, and rank $\left(W_{1}\right)=N$, where $W_{1}$ is any unknown general error.

The model (3.1) can be expressed as

$$
x_{u}=y_{u}+\sigma v=\beta_{0}+\sum_{i=1}^{k} \beta_{i} z_{u i}+\sum_{i \leq j}^{k} \beta_{i j} z_{u i} z_{u j}+\xi_{u}, \quad 1 \leq u \leq N, \quad \text { or, } \mathbf{X}=Z \boldsymbol{\beta}+\boldsymbol{\xi},
$$

considering $\sigma$ to be known for the time being, $v$ is known as Euler's constant, where $\xi_{u}=\sigma h_{1 u}+$ $\sigma v+e_{u}$ is the composite error (Das, Kim and Park, 2015). Note that $x_{u}=y_{u}+\sigma v$, and $\mathbf{X}=$ $\left(x_{1}, x_{2}, \ldots, x_{N}\right)^{\prime}$, is the vector of recorded origin changed natural logarithm of lifetime observations, $\boldsymbol{\beta}=\left(\beta_{0}, \beta_{11}, \ldots, \beta_{k k}, \beta_{1}, \ldots, \beta_{k}, \beta_{12}, \ldots, \beta_{(k-1) k}\right)^{\prime}$ is the vector of regression coefficients of order $\left(\begin{array}{c}k+2 \\ 2\end{array}\right) \times$ $1, Z=\left(\mathbf{1}: Z_{1}\right)$ is the design matrix, $Z_{1}=\left(\mathbf{z}_{1} \otimes_{1} \mathbf{z}_{1}, \ldots, \mathbf{z}_{k} \otimes_{1} \mathbf{z}_{k}, \mathbf{z}_{1}, \ldots, \mathbf{z}_{k}, \mathbf{z}_{1} \otimes_{1} \mathbf{z}_{2}, \ldots, \mathbf{z}_{k-1} \otimes_{1} \mathbf{z}_{k}\right)$, $\mathbf{z}_{i}=\left(z_{1 i}, \ldots, z_{N i}\right)^{\prime}, \quad 1 \leq i \leq k, \mathbf{z}_{i} \otimes_{1} \mathbf{z}_{j}=\left(z_{1 i} z_{1 j}, \ldots, z_{N i} z_{N j}\right)^{\prime} 1 \leq i, j \leq k$, and $\boldsymbol{\xi}=\left(\xi_{1}, \xi_{2}, \ldots, \xi_{N}\right)^{\prime}$. Then $E\left(\xi_{u}\right)=0, \operatorname{Var}\left(\xi_{u}\right)=\left(\sigma^{2} \pi^{2} / 6+\sigma_{1}^{2}\right)=\sigma_{2}^{2}, \operatorname{Cov}\left(\xi_{u}, \xi_{u^{\prime}}\right)=\operatorname{Cov}\left(e_{u}, e_{u^{\prime}}\right), \quad E(\boldsymbol{\xi})=\mathbf{0}, \operatorname{Dis}(\boldsymbol{\xi})=$ $\left(\sigma^{2} \pi^{2} / 6\right) I_{N}+\sigma_{1}^{2} W_{1}=\sigma_{2}^{2} W$ say, and ' $W$ ' is the composite correlated error structure (Das, Kim and Park, 2015), where,

$$
W=\left(\begin{array}{ccccc}
1 & \frac{\operatorname{Cov}\left(e_{1}, e_{2}\right)}{\sigma_{2}^{2}} & \frac{\operatorname{Cov}\left(e_{1}, e_{3}\right)}{\sigma_{2}^{2}} & \ldots & \frac{\operatorname{Cov}\left(e_{1}, e_{N}\right)}{\sigma_{2}^{2}} \\
\frac{\operatorname{Cov}\left(e_{2}, e_{1}\right)}{\sigma_{2}^{2}} & 1 & \frac{\operatorname{Cov}\left(e_{2}, e_{3}\right)}{\sigma_{2}^{2}} & \ldots & \frac{\operatorname{Cov}\left(e_{2}, e_{N}\right)}{\sigma_{2}^{2}} \\
\vdots & \ldots & \ldots & \ldots & \vdots \\
\frac{\operatorname{Cov}\left(e_{N}, e_{1}\right)}{\sigma_{2}^{2}} & \frac{\operatorname{Cov}\left(e_{N}, e_{2}\right)}{\sigma_{2}^{2}} & \frac{\operatorname{Cov}\left(e_{N}, e_{3}\right)}{\sigma_{2}^{2}} & \ldots & 1
\end{array}\right) .
$$

For the deriving model (3.2) under the Weibull distribution, we assume $\sigma$ to be known. Practically, $\sigma$ is unknown, and it is replaced by its estimate $\hat{\sigma}$ based on the original lifetime observations, where lifetime $T_{1}$ follows the Weibull distribution as in (1.1). For all computations, we consider, $x_{u}=$ $y_{u}+\hat{\sigma} v, \quad u=1,2, \ldots, N$.

As in (1.2), for $\delta_{1}=1$, implying $\sigma=1$, the second-order correlated lifetime model for exponential distribution is identical to (3.2), with $x_{u}=y_{u}+v, \xi_{u}=h_{1 u}+v+e_{u}, E\left(\xi_{u}\right)=0$, and $\operatorname{Var}\left(\xi_{u}\right)=\pi^{2} / 6+\sigma_{1}^{2}$ $=\sigma_{2}^{2}, u=1,2 \ldots, N, \quad E(\boldsymbol{\xi})=\mathbf{0}, \operatorname{Dis}(\boldsymbol{\xi})=\left(\pi^{2} / 6\right) I_{N}+\sigma_{1}^{2} W_{1}=\sigma_{2}^{2} W$, where $W$ is as in (3.2).

For the gamma distribution as in (1.3), we have the same second-order correlated lifetime model as in (3.2) with $x_{u}=y_{u}-\alpha_{0}, \xi_{u}=h_{2 u}-\alpha_{0}+e_{u}, \alpha_{0}=E\left(h_{2 u}\right), E\left(\xi_{u}\right)=0$, and $\operatorname{Var}\left(\xi_{u}\right)=\alpha_{2}^{2}+\sigma_{1}^{2}$ $=\sigma_{2}^{2}, u=1,2 \ldots, N, \quad E(\boldsymbol{\xi})=\mathbf{0}, \operatorname{Dis}(\boldsymbol{\xi})=\alpha_{2}^{2} I_{N}+\sigma_{1}{ }^{2} W_{1}=\sigma_{2}^{2} W$, assuming $\alpha_{0}$ to be known for the time being, where $\alpha_{2}^{2}=\operatorname{Var}\left(h_{2}\right), h_{2}$ follows the log-gamma distribution as in (1.3) and $W$ is as in (3.2). The same assumptions as $h_{1 u}$ are satisfied by $h_{2 u}$. For all theoretical derivations with the gamma distribution, we assume $\alpha_{0}$ is known. Practically $\alpha_{0}$ is unknown, and it is replaced by its estimate $\hat{\alpha}_{0}$ 
with the original lifetime observations, where lifetime $T_{1}$ follows gamma distribution as in (1.3). It is considered that $x_{u}=y_{u}-\hat{\alpha}_{0}, u=1,2, \ldots, N$.

For the lognormal lifetime distribution as in (1.4), we have the same model as in (3.2) with $x_{u}=y_{u}$, $\xi_{u}=\delta_{2} \tau_{u}+e_{u}, E\left(\xi_{u}\right)=0$ and $\operatorname{Var}\left(\xi_{u}\right)=\delta_{2}^{2}+\sigma_{1}^{2}=\sigma_{2}^{2}, u=1,2 \ldots, N, E(\boldsymbol{\xi})=\mathbf{0}, \operatorname{Dis}(\boldsymbol{\xi})=\delta_{2}^{2} I_{N}+\sigma_{1}^{2} W_{1}=$ $\sigma_{2}^{2} W$, where $\tau$ follows the standard normal distribution and $W$ is as in (3.2). The same assumptions as $h_{1 u}$ are satisfied by $\tau_{u}$.

Note that for the considered four lifetime distributions, the second-order response surface model is same as in (3.2), but only the unknown composite error $(\xi)$ and its dispersion structure are different for different lifetime distributions. Also, $\operatorname{Dis}(\xi)=\sigma_{2}^{2} I_{N}$, if $\operatorname{Cov}\left(e_{i}, e_{j}\right)=0$ for all $i \neq j, W=I_{N}$. Generally, $W$ is unknown but for all the calculations $W$ is assumed to be known. In practice, however, $W$ contains a number of unknown parameters, and in the calculations which follow, the expressions for $W$ and $W^{-1}$ are replaced by those obtained after replacing the unknown parameters with appropriate estimates or some assumed values. Under this situation, with the appropriate selection of the experimental levels $z_{u i}$ 's, $Z=\left(\left(z_{u i}\right), 1 \leq u \leq N, 1 \leq i \leq k\right)$, the design matrix is the most important part for getting maximum information regarding the unknown regression parameters. This situation is little studied for the second-order response surface models in Das (1997). The present article aims to develop robust second-order rotatable designs for the considered four lifetime distributions under the intra-class and inter-class correlation structure of $W_{1}$. It is shown herein that the derived secondorder rotatable designs are independent of the considered four lifetime distributions, as well as the correlation parameter values in $W_{1}$, and it only depends on the pattern of the correlation structure $W_{1}$. Therefore, the derived designs are termed as invariant (free of these considered four lifetime distributions) and robust (free of correlation parameter values) second-order rotatable designs.

\section{Second-order rotatability conditions with correlated errors}

For the second-order correlated lifetime model (3.2), we assume $\left(Z^{\prime} W^{-1} Z\right)$ is positive definite for known $W$, which is the best linear unbiased estimator of $\boldsymbol{\beta}$ is $\hat{\boldsymbol{\beta}}=\left(Z^{\prime} W^{-1} Z\right)^{-1}\left(Z^{\prime} W^{-1} \mathbf{X}\right)$ with,

$$
\operatorname{Dis}(\hat{\boldsymbol{\xi}})=\left(Z^{\prime} W^{-1} Z\right)^{-1}=\left(\begin{array}{lll}
A & B & C \\
B^{\prime} & P & Q \\
C^{\prime} & Q^{\prime} & R
\end{array}\right)_{\left(\begin{array}{c}
k+2 \\
2
\end{array}\right) \times\left(\begin{array}{c}
\left(\begin{array}{c}
k+2 \\
2
\end{array}\right) \\
\end{array}\right.}
$$

where $A, P$ and $R$ are all symmetric matrices given by

$$
A_{(k+1) \times(k+1)}=\left(\begin{array}{cccccc}
f_{00} & f_{0.11} & f_{0.22} & \cdots & f_{0 . k k} \\
f_{11.0} & & \left(f_{i i . j j}\right), & 1 \leq i, j \leq k & & \\
\vdots & \ldots & \ldots & \ldots & \vdots \\
f_{k k .0} & \cdots & \ldots & \ldots & \ldots
\end{array}\right),
$$

$f_{00}=\mathbf{1}^{\prime} W^{-1} \mathbf{1}, f_{0 . j j}=\mathbf{1}^{\prime} W^{-1}\left(\mathbf{z}_{j} \otimes_{1} \mathbf{z}_{j}\right)=\left(\mathbf{z}_{j} \otimes_{1} \mathbf{z}_{j}\right)^{\prime} W^{-1} \mathbf{1}=f_{j j .0}, 1 \leq j \leq k, f_{i i . j j}=\left(\mathbf{z}_{i} \otimes_{1} \mathbf{z}_{i}\right)^{\prime} W^{-1}\left(\mathbf{z}_{j} \otimes_{1} \mathbf{z}_{j}\right)$, $1 \leq i, j \leq k$,

$$
\begin{aligned}
& B_{(k+1) \times k}=\left(\begin{array}{ccc}
f_{0.1} & f_{0.2} \ldots & f_{0 . k} \\
& \left(f_{i i . j}\right)_{k \times k}, \quad 1 \leq i, j \leq k
\end{array},\right. \\
& f_{0 . j}=\mathbf{1}^{\prime} W^{-1} \mathbf{z}_{j}, \quad f_{i i . j}=\left(\mathbf{z}_{i} \otimes_{1} \mathbf{z}_{i}\right)^{\prime} W^{-1} \mathbf{z}_{j}, \quad 1 \leq i, j \leq k, \\
& C_{(k+1) \times\left(\begin{array}{l}
k \\
2
\end{array}\right)}=\left(\begin{array}{ccc}
f_{0.12} & f_{0.13} \ldots & f_{0 .(k-1) k} \\
& \left(f_{i i . j l}\right)_{k \times\left(\begin{array}{c}
k \\
2
\end{array}\right)}, \quad 1 \leq i, j<l \leq k &
\end{array}\right),
\end{aligned}
$$




$$
\begin{aligned}
f_{0 . j l} & =\mathbf{1}^{\prime} W^{-1}\left(\mathbf{z}_{j} \otimes_{1} \mathbf{z}_{l}\right), \quad f_{i i . j l}=\left(\mathbf{z}_{i} \otimes_{1} \mathbf{z}_{i}\right)^{\prime} W^{-1}\left(\mathbf{z}_{j} \otimes_{1} \mathbf{z}_{l}\right), \quad 1 \leq i \leq k, 1 \leq j<l \leq k, \\
P_{k \times k} & =\left(\left(f_{i . j}\right)\right), f_{i . j}=\mathbf{z}_{i}^{\prime} W^{-1} \mathbf{z}_{j}, \quad 1 \leq i, j \leq k, \\
Q_{k \times\left(\begin{array}{c}
k \\
2
\end{array}\right)} & =\left(\left(f_{i . j l}\right)\right), \quad f_{i . j l}=\mathbf{z}_{i}^{\prime} W^{-1}\left(\mathbf{z}_{j} \otimes_{1} \mathbf{z}_{l}\right), \quad 1 \leq i, j<l \leq k, \\
R_{\left(\begin{array}{c}
k \\
2
\end{array}\right) \times\left(\begin{array}{l}
k \\
2
\end{array}\right)} & =\left(\left(f_{i j . l t}\right)\right), f_{i j . l t}=\left(\mathbf{z}_{i} \otimes_{1} \mathbf{z}_{j}\right)^{\prime} W^{-1}\left(\mathbf{z}_{l} \otimes_{1} \mathbf{z}_{t}\right), \quad 1 \leq i<j \leq k, \quad 1 \leq l<t \leq k .
\end{aligned}
$$

Note that $f_{0 . j}=f_{j .0}, f_{0 . j j}=f_{j j .0}, f_{0 . j l}=f_{j l .0}, f_{i . j}=f_{j . i}, f_{i i . j}=f_{j . i i}, f_{i . j l}=f_{j l . i}, f_{i i . j j}=f_{j j . i i}, f_{i j . l t}=$ $f_{l t . i j}$. In the inverse matrix $\left(Z^{\prime} W^{-1} Z\right)^{-1}$, the element corresponding to $f_{m}$ in $\left(Z^{\prime} W^{-1} Z\right)$ is denoted by $f^{m}$ for all ' $m$ ' included in the preceding expressions.

Following Das (1997), the necessary and sufficient conditions of second-order rotatability for the model (3.2) (with correlation structure $W$ ) are the following,

(I) (i) $f_{0 . j}=f_{0 . j l}=0, \quad 1 \leq j<l \leq k$,

(ii) $f_{i . j}=0, \quad 1 \leq i, \quad j \leq k, \quad i \neq j$,

(iii) (1) $f_{i i . j}=0, \quad 1 \leq i \leq k, \quad 1 \leq j \leq k$,

(2) $f_{i . j l}=0, \quad 1 \leq i \leq k, \quad 1 \leq j<l \leq k$,

(3) $f_{i i . j l}=0, \quad 1 \leq i \leq k, \quad 1 \leq j<l \leq k$,

(4) $f_{i j . l t}=0, \quad 1 \leq i<j \leq k, \quad 1 \leq l<t \leq k, \quad(i, j) \neq(l, t)$,

(II) (i) $f_{0 . j j}=$ constant $=a_{0}(>0), \quad 1 \leq j \leq k$,

(ii) $f_{i . i}=$ constant $=\frac{1}{e}(>0), \quad 1 \leq i \leq k$,

(iii) $f_{\text {ii.ii }}=$ constant $=\left(\frac{2}{c}+d\right)(>0), \quad 1 \leq i \leq k$,

(III) (i) $f_{i i . j j}=$ constant $=d(>0), \quad 1 \leq i, \quad j \leq k, \quad i \neq j$,

(ii) $f_{i j . i j}=$ constant $=\frac{1}{c}(>0), \quad 1 \leq i<j \leq k$,

(IV)

$$
f_{i i . i i}=2 f_{i j . i j}+f_{i i . j j}, \quad 1 \leq i<j \leq k .
$$

The non-singularity condition of correlated second-order rotatable design is

(V)

$$
\frac{2}{c}+k\left(d-\frac{a_{0}^{2}}{f_{00}}\right)>0
$$

where $f_{00}$ and $f$ 's are in (4.1), and $a_{0}, 1 / c$, and $d$ are as in (4.2).

Following Das (1997), the variance of the estimated response at $\mathbf{z}$ (variance function) of a robust second-order rotatable design is given by,

$$
\operatorname{Var}\left(\hat{x}_{\mathbf{z}}\right)=f^{00}+\left(2 a_{1}+e\right) r^{2}+\left(\frac{c}{2}+d_{1}\right) r^{4},
$$


which is a function of $r^{2}=\sum_{i=1}^{k} z_{i}^{2}$, where

$$
f^{00}=\frac{\left(\frac{2}{c}+k d\right)}{\left\{f_{00}\left(\frac{2}{c}+k d\right)-k a_{0}^{2}\right\}}, \quad a_{1}=\frac{-a_{0}}{\left\{f_{00}\left(\frac{2}{c}+k d\right)-k a_{0}^{2}\right\}}, \quad d_{1}=\frac{c\left(a_{0}^{2}-d f_{00}\right)}{2\left\{f_{00}\left(\frac{2}{c}+k d\right)-k a_{0}^{2}\right\}},
$$

and $f_{00}, c, d, e$ are as in (4.2).

Note that a design ' $d$ ' of $k$ factors for the correlated lifetime model (3.2) remains second-order rotatable for all the variance-covariance matrices belonging to a well defined class $W_{0}=W$ positive definite: $W_{N \times N}$ defined by a particular correlation structure possessing a definite pattern is called a robust second-order rotatable design (RSORD), with reference to the variance-covariance class $W_{0}$.

\section{RSORDs for correlated lifetime models under intra and inter-class error structures}

The present section derives the necessary and sufficient conditions for second-order rotatability following Das (1997) for lognormal, gamma, exponential, and Weibull lifetime distributions under intraclass and inter-class error variance-covariance structure $W_{1}$. These two correlation structures are very similar, where the inter-class structure is an extension of intra-class structure. RSORDs are derived for both the correlation structures. Practically, inter-class structure is mostly observed in many data sets.

\subsection{Intra-class correlation structure}

The simplest dispersion matrix, or errors variance-covariance structure, is known as 'intra-class' or 'uniform' correlation structure. This dispersion structure occurs if any two observational errors have the identical correlation coefficient $\left(\rho^{*}\right)$ and each with identical variance $\left(\sigma_{1}^{2}\right)$. This arises if all the observations are examined under identical conditions, or from the same run in a batch, or from the same furnace. It is presented by,

$$
\operatorname{Dis}(\mathbf{e})=\sigma_{1}^{2}\left[\left(1-\rho^{*}\right) I_{N}+\rho^{*} E_{N \times N}\right]=\sigma_{1}^{2} W_{1}\left(\rho^{*}\right),
$$

where $\sigma_{1}>0,-(N-1)^{-1}<\rho^{*}<1, I_{N}$ is an identity matrix of order $N$, and $E_{N \times N}$ is a matrix of order $N \times N$ with all elements 1 (unity). The combined error dispersion structure $\left(\sigma_{2}^{2} W\right)$ as in (3.2) reduces to the intra-class structure $\sigma_{2}^{2} W_{1}(\rho)$ as in (5.1), where $\rho=\sigma_{1}^{2} / \sigma_{2}^{2} \rho^{*}$ and $\sigma_{2}^{2}=\left(\sigma^{2} \pi^{2} / 6+\sigma_{1}^{2}\right)$ for Weibull distribution, $\sigma_{2}^{2}=\left(\pi^{2} / 6+\sigma_{1}^{2}\right)$ for exponential distribution, $\sigma_{2}^{2}=\left(\alpha_{2}^{2}+\sigma_{1}^{2}\right)$ for gamma distribution and $\sigma_{2}^{2}=\left(\delta_{2}^{2}+\sigma_{1}^{2}\right)$ for lognormal distribution.

For the second-order model (3.2) (for all the four considered lifetime distributions) with errors having intra-class structure $\sigma_{1}^{2} W_{1}\left(\rho^{*}\right)$ as in (5.1), the simplified correlated second-order rotatability and non-singularity conditions (4.2) and (4.3) give the following designs along with all necessary and sufficient conditions.

The necessary and sufficient conditions of a robust second-order rotatable design (for all the four considered lifetime distributions) under the intra-class error structure (5.1), for all values of $\rho \in \sigma_{2}^{2} W(\rho)$ as in (5.1), in the model (3.2) are (for all $1 \leq i, j \leq k$ )

(I) $\sum_{u=1}^{N} z_{u i_{1}}^{\gamma_{1}} z_{u i_{2}}^{\gamma_{2}} z_{u i_{3}}^{\gamma_{3}} z_{u i_{4}}^{\gamma_{4}}=0, \quad$ for any $\gamma_{i}$ is odd and $\sum_{i=1}^{4} \gamma_{i} \leq 4$,

(II) (i) $\sum_{u=1}^{N} z_{u i}^{2}=$ constant , (ii) $\sum_{u=1}^{N} z_{u i}^{4}=$ constant

(III) $\sum_{u=1}^{N} z_{u i}^{2} z_{u j}^{2}=$ constant,$\quad i \neq j$, 
(IV) $\sum_{u=1}^{N} z_{u i}^{4}=3 \sum_{u=1}^{N} z_{u i}^{2} x_{u j}^{2}, \quad i \neq j$,

(V)

$$
\frac{\lambda_{4}}{\lambda_{2}^{2}}>\frac{k}{k+2} \quad \text { where, } \quad N \lambda_{2}=\sum_{u=1}^{N} z_{u i}^{2} \quad \text { and } \quad 3 N \lambda_{4}=\sum_{u=1}^{N} z_{u i}^{4} .
$$

The above conditions (I)-(V) as in (5.2) are independent of intra-class correlation coefficient $\rho$ and are the same as the necessary and sufficient conditions of usual independent errors second-order rotatable designs (SORDs). Therefore, usual SORDs are invariant (independent of any one of the considered four lifetime distributions) RSORDs for the intra-class error structure (5.1).

\subsection{Inter-class correlation structure}

Inter-class error dispersion matrix, or errors correlation structure is often observed in practice. For instance, products from different furnaces, or in batch products, where it is noted that observations are grouped into some batches such that within each batch there is the same intra-class correlation and between batches there is no correlation. Let ' $m$ ' be the number of groups and ' $n$ ' be the number of observations in each group. Similar situations can indicate inter-class correlation structure in a production system, which is displayed as follows.

$$
\operatorname{Dis}(\mathbf{e})=\sigma_{1}^{2}\left[I_{m} \otimes A\right]=\sigma_{1}^{2} W_{1}\left(\rho^{*}, 0\right),
$$

where $\otimes$ denotes Kronecker product, $A=\left(1-\rho^{*}\right) I_{n}+\rho^{*} E_{n \times n}$ (the intra-class structure as in (5.1)), $E_{n \times n}$ is as in (5.1) and $N=m n$. For the error structure (5.3), the composite error structure $\left(\sigma_{2}^{2} W\right)$ as in (3.2) reduces to the inter-class structure $\sigma_{2}^{2} W_{1}(\rho, 0)$ as in (5.3), where $\rho=\sigma_{1}{ }^{2} / \sigma_{2}{ }^{2} \rho^{*}$ and $\sigma_{2}{ }^{2}$ is as in (5.1), and its values are different for the different distributions.

For all the considered four lifetime distributions with second-order model (3.2), the second-order rotatability (4.2) and non-singularity (4.3) conditions under the inter-class structure (5.3) reduce to

$$
\begin{aligned}
& \text { (i) } f_{0 . j}=0 \Leftrightarrow \sum_{u=1}^{N} z_{u j}=0, \quad 1 \leq j \leq k, f_{0 . j l}=0 \Leftrightarrow \sum_{u=1}^{N} z_{u j} z_{u l}=0, \quad 1 \leq j<l \leq k, \\
& \text { (ii) } f_{i . j}=0 \Leftrightarrow \frac{1}{1-\rho} \sum_{u=1}^{N} z_{u i} z_{u j}-\frac{\rho}{(1-\rho)\{1+(n-1) \rho\}}\left\{\sum_{u=1}^{n} z_{u i} \sum_{u=1}^{n} z_{u j}+\cdots+\right. \\
& \left.\sum_{u=n(m-1)+1}^{m n} z_{u i} \sum_{u=n(m-1)+1}^{m n} z_{u j}\right\}=0, \quad 1 \leq i, j \leq k, i \neq j,
\end{aligned}
$$

(iii) (1) $f_{i i . j}=0 \Leftrightarrow \frac{1}{1-\rho} \sum_{u=1}^{N} z_{u i}^{2} z_{u j}-\frac{\rho}{(1-\rho)\{1+(n-1) \rho\}}\left\{\sum_{u=1}^{n} z_{u i}^{2} \sum_{u=1}^{n} z_{u j}+\cdots+\right.$

$$
\left.\sum_{u=n(m-1)+1}^{m n} z_{u i}^{2} \sum_{u=n(m-1)+1}^{m n} z_{u j}\right\}=0, \quad 1 \leq i \leq k, 1 \leq j \leq k
$$

(2) $f_{i j . l}=0 \Leftrightarrow \frac{1}{1-\rho} \sum_{u=1}^{N} z_{u i} z_{u j} z_{u l}-\frac{\rho}{(1-\rho)\{1+(n-1) \rho\}}\left\{\sum_{u=1}^{n} z_{u i} z_{u j} \sum_{u=1}^{n} z_{u l}+\cdots+\right.$ $\left.\sum_{u=n(m-1)+1}^{m n} z_{u i} z_{u j} \sum_{u=n(m-1)+1}^{m n} z_{u l}\right\}=0, \quad 1 \leq i<j \leq k, \quad 1 \leq l \leq k$, 
(3) $f_{i i . j l}=0 \Leftrightarrow \frac{1}{1-\rho} \sum_{u=1}^{N} z_{u i}^{2} z_{u j} z_{u l}-\frac{\rho}{(1-\rho)\{1+(n-1) \rho\}}\left\{\sum_{u=1}^{n} z_{u i}^{2} \sum_{u=1}^{n} z_{u j} z_{u l}+\cdots+\right.$ $\left.\sum_{u=n(m-1)+1}^{m n} z_{u i}^{2} \sum_{u=n(m-1)+1}^{m n} z_{u j} z_{u l}\right\}=0, \quad 1 \leq i \leq k, 1 \leq j<l \leq k$,

(4) $f_{i j . l t}=0 \Leftrightarrow \frac{1}{(1-\rho)} \sum_{u=1}^{N} z_{u i} z_{u j} z_{u l} z_{u t}-\frac{\rho}{(1-\rho)\{1+(n-1) \rho\}}\left\{\sum_{u=1}^{n} z_{u i} z_{u j} \sum_{u=1}^{n} z_{u l} z_{u t}+\cdots+\right.$ $\left.\sum_{u=n(m-1)+1}^{m n} z_{u i} z_{u j} \sum_{u=n(m-1)+1}^{m n} z_{u l} z_{u t}\right\}=0, \quad 1 \leq i<j \leq k, 1 \leq l<t \leq k, \quad(i, j) \neq(l, t)$

(II) (i) $f_{0 . j j}=$ constant $\Leftrightarrow\left\{\sigma^{2}(1+(n-1) \rho)\right\}^{-1} \sum_{u=1}^{N} z_{u j}^{2}=a_{0}(>0), \quad 1 \leq j \leq k$,

(ii) $f_{i . i}=$ constant $\Leftrightarrow \sigma^{-2}\left[\frac{1}{(1-\rho)} \sum_{u=1}^{N} z_{u i}^{2}-\frac{\rho}{(1-\rho)\{1+(n-1) \rho\}}\left\{\left(\sum_{u=1}^{n} z_{u i}\right)^{2} \cdots+\right.\right.$ $\left.\left.\left(\sum_{u=n(m-1)+1}^{m n} z_{u i}\right)^{2}\right\}\right]=\frac{1}{e}(>0), 1 \leq i \leq k$,

(iii) $f_{i i . i i}=$ constant $\Leftrightarrow \sigma^{-2}\left[\frac{1}{(1-\rho)} \sum_{u=1}^{N} z_{u i}^{4}-\frac{\rho}{(1-\rho)\{1+(n-1) \rho\}}\left\{\left(\sum_{u=1}^{n} z_{u i}^{2}\right)^{2}+\cdots+\right.\right.$ $\left.\left.\left(\sum_{u=n(m-1)+1}^{m n} z_{u i}^{2}\right)^{2}\right\}\right]=\left(\frac{2}{c}+d\right)(>0), 1 \leq i \leq k$,

(III) (i) $f_{i i . j j}=$ constant $\Leftrightarrow \sigma^{-2}\left[\frac{1}{(1-\rho)} \sum_{u=1}^{N} z_{u i}^{2} z_{u j}^{2}-\frac{\rho}{(1-\rho)\{1+(n-1) \rho\}}\left\{\sum_{u=1}^{n} z_{u i}^{2} \sum_{u=1}^{n} z_{u j}^{2}+\cdots+\right.\right.$ $\left.\left.\sum_{u=n(m-1)+1}^{m n} z_{u i}^{2} \sum_{u=n(m-1)+1}^{m n} z_{u j}^{2}\right\}\right]=d(>0), \quad 1 \leq i, j \leq k, i \neq j$,

(ii) $f_{i j . i j}=$ constant $\Leftrightarrow \sigma^{-2}\left[\frac{1}{(1-\rho)} \sum_{u=1}^{N} z_{u i}^{2} z_{u j}^{2}-\frac{\rho}{(1-\rho)\{1+(n-1) \rho\}}\left\{\left(\sum_{u=1}^{n} z_{u i} z_{u j}\right)^{2}+\cdots+\right.\right.$ $\left.\left.+\left(\sum_{u=n(m-1)+1}^{m n} z_{u i} z_{u j}\right)^{2}\right\}\right]=\frac{1}{c}(>0), \quad 1 \leq i<j \leq k$,

(IV) $f_{i i . i i}=2 f_{i j . i j}+f_{i i . j j} ; 1 \leq i<j \leq k$,

(V)

$$
\frac{2}{c}+k\left(d-\frac{a_{0}^{2}}{f_{00}}\right)>0
$$

where $f_{i i . i i}, f_{i i . j j}$ and $f_{i j . i j}$ are as in (II) (iii) and (III) (i), (ii), $f_{00}=N / 1+(n-1) \rho$ and $1 / c, d, a_{0}$ are as in (5.4). 


\subsection{Construction of RSORDs under inter-class structure}

Method I: For all the considered four lifetime distributions with model (3.2) and errors having the inter-class structure (5.3), RSORDs can be constructed from ' $m$ ' usual SORDs (each with $k$ factors and $n$ runs) by arranging each SORD as the design points in each group with ' $n$ ' runs, such that the design points in each group satisfy the second-order rotatable structure in the usual sense (with uncorrelated errors). Then all these ' $m$ ' usual SORDs from ' $m$ ' groups form a RSORD for this situation.

(a) It can be readily examined that (5.4) holds for these designs.

(b) For a non-singularity condition, the following design parameters can be noted.

$$
\begin{aligned}
& a_{0}=\frac{m n \lambda_{2}}{\sigma^{2}\{1+(n-1) \rho\}}=\frac{N \lambda_{2}}{\sigma^{2}\{1+(n-1) \rho\}}, \quad \frac{1}{e}=\frac{N \lambda_{2}}{\sigma^{2}(1-\rho)}, d=\frac{1}{\sigma^{2}}\left[\frac{N \lambda_{4}}{(1-\rho)}-\frac{m \rho\left(n \lambda_{2}\right)^{2}}{(1-\rho)\{1+(n-1) \rho\}}\right], \frac{1}{c}=\frac{1}{\sigma^{2}}\left[\frac{N \lambda_{4}}{(1-\rho)}\right], \\
& f_{00}=\frac{N}{\sigma^{2}\{1+(n-1) \rho\}} .
\end{aligned}
$$

Using these design parameters, the non-singularity condition $2 / c+k\left(d-a_{0}^{2} / f_{00}\right)$ in (5.4) simplifies to,

$$
\frac{\lambda_{4}}{\lambda_{2}^{2}}>\frac{k}{k+2},
$$

which is the non-singularity condition for each set of observations of ' $m$ ' sets. Therefore, the designs described by Method I satisfy the non-singularity condition for all values of $\rho$.

Method II: For all the considered four lifetime distributions with model (3.2) and errors having the inter-class structure (5.3), RSORDs can be constructed as follow. Let $D_{0}: k \times 2^{p}$ be a design matrix which is obtained from the $1 / 2^{k-p}$ fraction of $2^{k}$-experiment with levels -1 and +1 , where the defining equation in obtaining the fraction does not include any main effect or interactions containing four or fewer factors (Resolution V). Suppose the array of treatment combinations is $L_{(k \times G)}=D_{0}$ with $G=$ $2^{p}$, where $L$ can be partitioned as $L_{(k \times G)}=\left[L_{1\left(k \times G_{0}\right)}: L_{2\left(k \times G_{0}\right)}: \ldots: L_{s\left(k \times G_{0}\right)}\right]$, where $s G_{0}=G$, and the inner product of any two distinct rows of $L_{i}$ is zero, $i=1,2, \ldots, s$.

Let us consider a matrix $H_{1}$ of order $(k+1) \times s$ written with elements -1 and +1 such that the inner product of every pair of rows of the matrix is 0 (zero). Based on the conditions of $H_{1}$, it can be arbitrarily assumed that the first row of $H_{1}$ consists of all +1 's. By deleting the first row from $H_{1}$, we get,

$$
H_{10}=\left(\begin{array}{ccccc}
h_{11} & h_{12} & h_{13} & \cdots & h_{1 s} \\
h_{21} & h_{22} & h_{23} & \cdots & h_{2 s} \\
\vdots & \cdots & \cdots & \cdots & \vdots \\
\vdots & \ldots & \cdots & \cdots & \vdots \\
h_{k 1} & h_{k 2} & h_{k 3} & \cdots & h_{k s}
\end{array}\right) .
$$

Suppose $D_{i}=\varsigma \operatorname{Diag}\left(h_{1 i}, h_{2 i}, \ldots, h_{k i}\right), F_{i}=\left[L_{i}: D_{i}\right], i=1,2, \ldots, s$ and $\mathrm{F}=\left(F_{1}: F_{2}: \ldots,: F_{s}\right)$. The $k$ rows of matrix $F$ form a RSORD under the inter-class structure (5.3) of $k$ factors with $m=s$ groups, with each group of size $n=\left(G_{0}+k\right)$ and number of design points, $N=s\left(G_{0}+k\right)=s G_{0}+s k$ $=G+s k=2^{p}+m k, s=m$ being the number of groups in which the observations are divided. Note that ' $s$ ' is determined from the condition (IV) of (5.4), and the design points which are obtained from the part $L_{i}$ of $F_{i}$ are from the appropriate fractional factorial $2^{k}$ experiment, are given by $D_{i}, i=1,2$, 
$\ldots, m$ are the so called star points. Thus, the overall design so constructed, forms a kind of central composite design usually constructed.

Obviously, in this design construction method, a factor point (obtained either from the appropriate fraction of a $2^{k}$ experiment or the star points) may have to be repeated. In certain situations, the repetition has to be accepted so that different parameters of the design match in order to give an overall rotatable design in the present context. The parts of the design belonging to the different groups do not give a SORD.

The RSORDs obtained by the Method II satisfy condition (I) of (5.4). The expressions (i), (ii) and (iii) in (II) of (5.4) simplify respectively to,

$$
\begin{aligned}
a_{0} & =\frac{\left(G_{0}+\varsigma^{2}\right) m}{\sigma^{2}\{1+(n-1) \rho\}}, \quad \frac{1}{e}=\frac{1}{\sigma^{2}}\left[\frac{\left(G_{0}+\varsigma^{2}\right) m}{1-\rho}-\frac{\rho \varsigma^{2} m}{(1-\rho)\{1+(n-1) \rho\}}\right] \\
\left(\frac{2}{c}+d\right) & =\frac{1}{\sigma^{2}}\left[\frac{\left(G_{0}+\varsigma^{4}\right) m}{1-\rho}-\frac{\rho\left(G_{0}+\varsigma^{2}\right)^{2} m}{(1-\rho)\{1+(n-1) \rho\}}\right]
\end{aligned}
$$

and they are the same for $i=1,2, \ldots, k$.

Note that $n=\left(G_{0}+k\right), N=m n=\left(G_{0}+k\right) m=G+m k=2^{p}+m k, \quad s=m$. Again, the expressions (i) and (ii) in (III) of (5.4) reduce respectively to,

$$
d=\frac{1}{\sigma^{2}}\left[\frac{G_{0} m}{1-\rho}-\frac{\rho\left(G_{0}+\varsigma^{2}\right)^{2} m}{(1-\rho)\{1+(n-1) \rho\}}\right], \frac{1}{c}=\frac{1}{\sigma^{2}}\left(\frac{G_{0} m}{1-\rho}\right),
$$

and they are the same for $1 \leq i<j \leq k$.

Note that ' $s$ ' is determined from (IV) of (5.4) (using the above results), in consideration of the following equation,

$$
2 \frac{G_{0} m}{1-\rho}+\left[\frac{G_{0} m}{1-\rho}-\frac{\rho\left(G_{0}+\varsigma^{2}\right)^{2} m}{(1-\rho)\{1+(n-1) \rho\}}\right]=\frac{\left(G_{0}+\varsigma^{4}\right) m}{1-\rho}-\frac{\rho\left(G_{0}+\varsigma^{2}\right)^{2} m}{(1-\rho)\{1+(n-1) \rho\}}
$$

or, $3 G_{0}=G_{0}+\varsigma^{4}$ or, $\varsigma=\left(2 G_{0}\right)^{1 / 4}$.

Thus, with $\varsigma=\left(2 G_{0}\right)^{1 / 4}$, rotatability conditions (5.4) are satisfied by the design points described by Method II for all $\rho$.

Using the above design parameters and noting $f_{00}=N / \sigma^{2}\{1+(n-1) \rho\}$, the expression $2 / c+k(d-$ $\left.a_{0}^{2} / f_{00}\right)$ simplifies to,

$$
\frac{m}{\sigma^{2}(1-\rho)}\left[(k+2) G_{0}-k \frac{\left(G_{0}+\varsigma^{2}\right)^{2}}{n}\right] .
$$

Thus, the non-singularity condition (V) in (5.4) reduces to

$$
\begin{aligned}
& (k+2) G_{0}-k \frac{\left(G_{0}+\varsigma^{2}\right)^{2}}{n}>0 \\
& \Rightarrow \frac{n G_{0}}{\left(G_{0}+\varsigma^{2}\right)^{2}}>\frac{k}{k+2} \\
& \Rightarrow \frac{G_{0}+k}{G_{0}+2\left\{1+\left(2 G_{0}\right)^{\frac{1}{2}}\right\}}>\frac{k}{k+2} .
\end{aligned}
$$


Therefore, the designs described by Method II satisfy the non-singularity condition (5.5) for all values of $\rho$ as $G_{0}$ is always greater than $k$. So, the designs derived by using Method II are RSORDs under the inter-class structure (5.3).

Note that the above Method II for constructing RSORDs is quite powerful as the original design $D_{o}$ can also be selected from different sources other than factorial experiments such as balanced incomplete block (BIB) designs, which have been utilized extensively by many authors in the construction of SORDs with uncorrelated errors. Construction of a RSORD with $k=7$ factors using BIB design with parameters $v=b=7, r=k=3, \lambda=1$ is described as follows. Let the symmetrical BIB design with parameters $v=b=7, r=k=3, \lambda=1$ be given as $(0,1,3),(1,2,4),(2,3,5),(3,4,6),(4,5,0)$, $(5,6,1),(6,0,2)$. Let us consider the 7 factors corresponding to the 7 treatments in the BIB design. Construct a $7 \times 8$ matrix $L_{i}, i=1,2, \ldots, 7$ from the $i^{\text {th }}$ block as follows. Suppose the treatments in the $i^{\text {th }}$ block of the design are $\left(i_{1}, i_{2}, i_{3}\right)$. Then the three rows of the $7 \times 8$ matrix $L_{i}$, which correspond to the factors represented by the treatments $i_{1}, i_{2}$ and $i_{3}$ of the block give a full factorial experiment with 3 factors each having two levels +1 and -1 . Let us write 0 as the level of every other factor in each of the columns. The $7 \times 8$ matrix of $-1,+1$ and 0 's thus obtained is called $L_{i}$. The following example illustrates the above method.

Example: Using the BIB design for Method II, let us consider an experimental design with seven factors, eight independent groups, each of fifteen observations i.e., $k=7, m=8, n=15$ and $\operatorname{Dis}(\mathbf{e})=$ $I_{8} \otimes W_{15 \times 15}(\rho), N=m n=120$. The level combinations of 7 factors in 8 groups are given.

Group totals for $\mathbf{x}_{i}$ 's are given in the following table.

Note that the design points within each group do not satisfy the usual SORD conditions, but the overall design forms a RSORD under the inter-class structure (5.3). In addition, the number of design points by Method II is less than the number of points by Method I design.

\section{Concluding remarks}

The present article considers the univariate response lifetime random variable having distributions such as gamma, lognormal, Weibull and exponential, which are often observed in practice. Regressor variables are considered in lifetime models for handling heterogeneity. First-order lifetime location-scale regression models with constant variance are extended to second-order correlated models. Mixed linear logarithms design of lifetime correlated models are derived from two random components, whereas one component is associated with the response lifetime distribution and the other is the error term. Second-order rotatable designs are derived under these four lifetime distributions with errors having intra and inter-class structures. For the intra-class error structure, usual SORDs are robust SORDs and invariant for these four lifetime distributions. For inter-class structure, two design construction methods have been suggested which give invariant robust SORDs.

Lifetime data sets can be homogeneous, or heterogeneous with lognormal, or gamma distributed (Das and Lee, 2009; Das and Park, 2012; Das, 2013; Das, Kim and Park, 2015). In industrial design of experiments, experimental units can not be treated as independent, as they produce correlation among observations via a repeated measures scenario as in split plot design (Myers et al., 2002). From a practical point of view, the current manuscript has considered correlated non-linear secondorder models (as the fitted model is non-linear, $\hat{t}_{1 u}=\exp \left(\hat{\beta}_{0}+\sum_{i=1}^{k} \hat{\beta}_{i} z_{u i}+\sum_{i \leq j}^{k} \beta_{i j} z_{u i} z_{u j}\right)$ ) for some useful lifetime distributions. Two errors correlation structures such as intra-class and inter-class have been considered in the report, while intra-class is a particular form of inter-class structure that is relevant in many practical situations such as blocked experiments and split-plot experiments (Goos and Jones, 2011). Under these situations, improved designs are orthogonally blocked designs, which 


\begin{tabular}{|c|c|c|c|c|c|c|c|c|c|c|c|c|c|c|c|}
\hline $\begin{array}{ll}\text { Group } 1 \\
\end{array}$ & 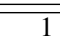 & 2 & 3 & 4 & 5 & $\overline{6}$ & 7 & 8 & 9 & 10 & 11 & 12 & 13 & 14 & 15 \\
\hline $\mathbf{x}_{1}$ & 0 & 0 & 0 & 0 & 0 & 0 & 0 & 0 & 2 & 0 & 0 & 0 & 0 & 0 & 0 \\
\hline $\mathbf{x}_{2}$ & -1 & -1 & 1 & 1 & -1 & -1 & 1 & 1 & 0 & 2 & 0 & 0 & 0 & 0 & 0 \\
\hline $\mathbf{x}_{3}$ & -1 & -1 & -1 & -1 & 1 & 1 & 1 & 1 & 0 & 0 & 2 & 0 & 0 & 0 & 0 \\
\hline $\mathbf{x}_{4}$ & 0 & 0 & 0 & 0 & 0 & 0 & 0 & 0 & 0 & 0 & 0 & 2 & 0 & 0 & 0 \\
\hline $\mathbf{x}_{5}$ & 1 & -1 & 1 & -1 & -1 & 1 & -1 & 1 & 0 & 0 & 0 & 0 & 2 & 0 & 0 \\
\hline $\mathbf{x}_{6}$ & 0 & 0 & 0 & 0 & 0 & 0 & 0 & 0 & 0 & 0 & 0 & 0 & 0 & 2 & 0 \\
\hline $\mathbf{x}_{7}$ & 0 & 0 & 0 & 0 & 0 & 0 & 0 & 0 & 0 & 0 & 0 & 0 & 0 & 0 & 2 \\
\hline Group 2 & 1 & 2 & 3 & 4 & 5 & 6 & 7 & 8 & 9 & 10 & 11 & 12 & 13 & 14 & 15 \\
\hline $\mathbf{x}_{1}$ & 0 & 0 & 0 & 0 & 0 & 0 & 0 & 0 & -2 & 0 & 0 & 0 & 0 & 0 & 0 \\
\hline $\mathbf{x}_{2}$ & 0 & 0 & 0 & 0 & 0 & 0 & 0 & 0 & 0 & 2 & 0 & 0 & 0 & 0 & 0 \\
\hline $\mathbf{x}_{3}$ & -1 & -1 & -1 & -1 & 1 & 1 & 1 & 1 & 0 & 0 & -2 & 0 & 0 & 0 & 0 \\
\hline $\mathbf{x}_{4}$ & 1 & -1 & -1 & 1 & 1 & -1 & -1 & 1 & 0 & 0 & 0 & 2 & 0 & 0 & 0 \\
\hline $\mathbf{x}_{5}$ & 0 & 0 & 0 & 0 & 0 & 0 & 0 & 0 & 0 & 0 & 0 & 0 & -2 & 0 & 0 \\
\hline $\mathbf{x}_{6}$ & 1 & 1 & -1 & -1 & -1 & -1 & 1 & 1 & 0 & 0 & 0 & 0 & 0 & 2 & 0 \\
\hline $\mathbf{x}_{7}$ & 0 & 0 & 0 & 0 & 0 & 0 & 0 & 0 & 0 & 0 & 0 & 0 & 0 & 0 & -2 \\
\hline Group 3 & 1 & 2 & 3 & 4 & 5 & 6 & 7 & 8 & 9 & 10 & 11 & 12 & 13 & 14 & 15 \\
\hline$\frac{1}{\mathbf{x}_{1}}$ & 0 & 0 & 0 & 0 & 0 & 0 & 0 & 0 & 2 & 0 & 0 & 0 & 0 & 0 & 0 \\
\hline $\mathbf{x}_{2}$ & 0 & 0 & 0 & 0 & 0 & 0 & 0 & 0 & 0 & -2 & 0 & 0 & 0 & 0 & 0 \\
\hline $\mathbf{x}_{3}$ & 0 & 0 & 0 & 0 & 0 & 0 & 0 & 0 & 0 & 0 & -2 & 0 & 0 & 0 & 0 \\
\hline $\mathbf{x}_{4}$ & 1 & -1 & -1 & 1 & 1 & -1 & -1 & 1 & 0 & 0 & 0 & 2 & 0 & 0 & 0 \\
\hline $\mathbf{x}_{5}$ & 1 & -1 & 1 & -1 & -1 & 1 & -1 & 1 & 0 & 0 & 0 & 0 & 2 & 0 & 0 \\
\hline $\mathbf{x}_{6}$ & 0 & 0 & 0 & 0 & 0 & 0 & 0 & 0 & 0 & 0 & 0 & 0 & 0 & -2 & 0 \\
\hline $\mathbf{x}_{7}$ & -1 & 1 & 1 & -1 & 1 & -1 & -1 & 1 & 0 & 0 & 0 & 0 & 0 & 0 & -2 \\
\hline Group 4 & 1 & 2 & 3 & 4 & 5 & 6 & 7 & 8 & 9 & 10 & 11 & 12 & 13 & 14 & 15 \\
\hline $\mathbf{x}_{1}$ & -1 & 1 & -1 & 1 & -1 & 1 & -1 & 1 & -2 & 0 & 0 & 0 & 0 & 0 & 0 \\
\hline $\mathbf{x}_{2}$ & 0 & 0 & 0 & 0 & 0 & 0 & 0 & 0 & 0 & -2 & 0 & 0 & 0 & 0 & 0 \\
\hline $\mathbf{x}_{3}$ & 0 & 0 & 0 & 0 & 0 & 0 & 0 & 0 & 0 & 0 & 2 & 0 & 0 & 0 & 0 \\
\hline $\mathbf{x}_{4}$ & 0 & 0 & 0 & 0 & 0 & 0 & 0 & 0 & 0 & 0 & 0 & 2 & 0 & 0 & 0 \\
\hline $\mathbf{x}_{5}$ & 1 & -1 & 1 & -1 & -1 & 1 & -1 & 1 & 0 & 0 & 0 & 0 & -2 & 0 & 0 \\
\hline $\mathbf{x}_{6}$ & 1 & 1 & -1 & -1 & -1 & -1 & 1 & 1 & 0 & 0 & 0 & 0 & 0 & -2 & 0 \\
\hline $\mathbf{x}_{7}$ & 0 & 0 & 0 & 0 & 0 & 0 & 0 & 0 & 0 & 0 & 0 & 0 & 0 & 0 & 2 \\
\hline Group 5 & 1 & 2 & 3 & 4 & 5 & 6 & 7 & 8 & 9 & 10 & 11 & 12 & 13 & 14 & 15 \\
\hline $\mathbf{x}_{1}$ & 0 & 0 & 0 & 0 & 0 & 0 & 0 & 0 & 2 & 0 & 0 & 0 & 0 & 0 & 0 \\
\hline $\mathbf{x}_{2}$ & -1 & -1 & 1 & 1 & -1 & -1 & 1 & 1 & 0 & 2 & 0 & 0 & 0 & 0 & 0 \\
\hline $\mathbf{x}_{3}$ & 0 & 0 & 0 & 0 & 0 & 0 & 0 & 0 & 0 & 0 & 2 & 0 & 0 & 0 & 0 \\
\hline $\mathbf{x}_{4}$ & 0 & 0 & 0 & 0 & 0 & 0 & 0 & 0 & 0 & 0 & 0 & -2 & 0 & 0 & 0 \\
\hline $\mathbf{x}_{5}$ & 0 & 0 & 0 & 0 & 0 & 0 & 0 & 0 & 0 & 0 & 0 & 0 & -2 & 0 & 0 \\
\hline $\mathbf{x}_{6}$ & 1 & 1 & -1 & -1 & -1 & -1 & 1 & 1 & 0 & 0 & 0 & 0 & 0 & -2 & 0 \\
\hline $\mathbf{x}_{7}$ & -1 & 1 & 1 & -1 & 1 & -1 & -1 & 1 & 0 & 0 & 0 & 0 & 0 & 0 & -2 \\
\hline Group 6 & 1 & 2 & 3 & 4 & 5 & 6 & 7 & 8 & 9 & 10 & 11 & 12 & 13 & 14 & 15 \\
\hline $\mathbf{x}_{1}$ & -1 & 1 & -1 & 1 & -1 & 1 & -1 & 1 & -2 & 0 & 0 & 0 & 0 & 0 & 0 \\
\hline $\mathbf{x}_{2}$ & 0 & 0 & 0 & 0 & 0 & 0 & 0 & 0 & 0 & 2 & 0 & 0 & 0 & 0 & 0 \\
\hline $\mathbf{x}_{3}$ & -1 & -1 & -1 & -1 & 1 & 1 & 1 & 1 & 0 & 0 & -2 & 0 & 0 & 0 & 0 \\
\hline $\mathbf{x}_{4}$ & 0 & 0 & 0 & 0 & 0 & 0 & 0 & 0 & 0 & 0 & 0 & -2 & 0 & 0 & 0 \\
\hline $\mathbf{x}_{5}$ & 0 & 0 & 0 & 0 & 0 & 0 & 0 & 0 & 0 & 0 & 0 & 0 & 2 & 0 & 0 \\
\hline $\mathbf{x}_{6}$ & 0 & 0 & 0 & 0 & 0 & 0 & 0 & 0 & 0 & 0 & 0 & 0 & 0 & -2 & 0 \\
\hline $\mathbf{x}_{7}$ & -1 & 1 & 1 & -1 & 1 & -1 & -1 & 1 & 0 & 0 & 0 & 0 & 0 & 0 & 2 \\
\hline
\end{tabular}

are $D$-optimal, and they are commonly used in quality engineering (Cheng, 1988). There are many more important errors correlation structures such as autocorrelated, tri-diagonal, compound symmetry, which are very realistic and useful. We propose to discuss these structures in our subsequent articles.

The developed designs in the article can be used to predict the optimal operating conditions that achieves the target mean value, while reducing the variance. Dual response surface (DRS) approach was introduced by Myers and Carter (1973) for this purpose, whereas second-order response surface 


\begin{tabular}{|c|c|c|c|c|c|c|c|c|c|c|c|c|c|c|c|}
\hline Group 7 & 1 & 2 & 3 & 4 & 5 & 6 & 7 & 8 & 9 & 10 & 11 & 12 & 13 & 14 & 15 \\
\hline $\mathbf{x}_{1}$ & -1 & 1 & -1 & 1 & -1 & 1 & -1 & 1 & 2 & 0 & 0 & 0 & 0 & 0 & 0 \\
\hline $\mathbf{x}_{2}$ & -1 & -1 & 1 & 1 & -1 & -1 & 1 & 1 & 0 & -2 & 0 & 0 & 0 & 0 & 0 \\
\hline $\mathbf{x}_{3}$ & 0 & 0 & 0 & 0 & 0 & 0 & 0 & 0 & 0 & 0 & -2 & 0 & 0 & 0 & 0 \\
\hline $\mathbf{x}_{4}$ & -1 & -1 & -1 & -1 & 1 & 1 & 1 & 1 & 0 & 0 & 0 & -2 & 0 & 0 & 0 \\
\hline $\mathbf{x}_{5}$ & 0 & 0 & 0 & 0 & 0 & 0 & 0 & 0 & 0 & 0 & 0 & 0 & -2 & 0 & 0 \\
\hline $\mathbf{x}_{6}$ & 0 & 0 & 0 & 0 & 0 & 0 & 0 & 0 & 0 & 0 & 0 & 0 & 0 & 2 & 0 \\
\hline $\mathbf{x}_{7}$ & 0 & 0 & 0 & 0 & 0 & 0 & 0 & 0 & 0 & 0 & 0 & 0 & 0 & 0 & 2 \\
\hline Group 8 & 1 & 2 & 3 & 4 & 5 & 6 & 7 & 8 & 9 & 10 & 11 & 12 & 13 & 14 & 15 \\
\hline $\mathbf{x}_{1}$ & 0 & 0 & 0 & 0 & 0 & 0 & 0 & 0 & -2 & 0 & 0 & 0 & 0 & 0 & 0 \\
\hline $\mathbf{x}_{2}$ & -1 & -1 & 1 & 1 & -1 & -1 & 1 & 1 & 0 & -2 & 0 & 0 & 0 & 0 & 0 \\
\hline $\mathbf{x}_{3}$ & -1 & -1 & -1 & -1 & 1 & 1 & 1 & 1 & 0 & 0 & 2 & 0 & 0 & 0 & 0 \\
\hline $\mathbf{x}_{4}$ & 0 & 0 & 0 & 0 & 0 & 0 & 0 & 0 & 0 & 0 & 0 & -2 & 0 & 0 & 0 \\
\hline $\mathbf{x}_{5}$ & 1 & -1 & 1 & -1 & -1 & 1 & -1 & 1 & 0 & 0 & 0 & 0 & 2 & 0 & 0 \\
\hline $\mathbf{x}_{6}$ & 0 & 0 & 0 & 0 & 0 & 0 & 0 & 0 & 0 & 0 & 0 & 0 & 0 & 2 & 0 \\
\hline $\mathbf{x}_{7}$ & 0 & 0 & 0 & 0 & 0 & 0 & 0 & 0 & 0 & 0 & 0 & 0 & 0 & 0 & -2 \\
\hline Group & & 1 & & 2 & & 3 & & $\overline{4}$ & & 5 & $\overline{6}$ & & 7 & & 8 \\
\hline $\mathbf{x}_{1}$ & & 2 & & -2 & & 2 & & -2 & & 2 & -2 & & 2 & & -2 \\
\hline $\mathbf{x}_{2}$ & & 2 & & 2 & & -2 & & -2 & & 2 & 2 & & -2 & & -2 \\
\hline $\mathbf{x}_{3}$ & & 2 & & -2 & & -2 & & 2 & & 2 & -2 & & -2 & & 2 \\
\hline $\mathbf{x}_{4}$ & & 2 & & 2 & & 2 & & 2 & & -2 & -2 & & -2 & & -2 \\
\hline $\mathbf{x}_{5}$ & & 2 & & -2 & & 2 & & -2 & & -2 & 2 & & -2 & & 2 \\
\hline $\mathbf{x}_{6}$ & & 2 & & 2 & & -2 & & -2 & & -2 & -2 & & 2 & & 2 \\
\hline $\mathbf{x}_{7}$ & & 2 & & -2 & & -2 & & 2 & & -2 & 2 & & 2 & & -2 \\
\hline
\end{tabular}

designs are used for separate modeling of mean and variance. For this purpose, the joint generalized linear models (JGLMs) have been proposed by Nelder and Lee (1991). Therefore, for both the DRS and JGLMs approaches, the present developed designs can be used to attain the specific target mean value, and simultaneously reduce the process variability.

\section{Acknowledgement}

The authors are very much indebted to the referees who have provided valuable comments to improve this paper. The work of Youngjo Lee was supported by a grant from the National Research Foundation of Korea (NRF) that was funded by the Korea government (MSIT) (No 2019R1A2C1002408).

\section{References}

Box GEP and Draper NR (2007). Response surfaces, Mixtures, and Ridge Analyses(Second edition), John Wiley and Sons, New York.

Box GEP and Hunter JS (1957). Multifactor experimental designs for exploring response surfaces, The Annals of Mathematical Statistics, 28, 195-241.

Cheng (1988). Some hidden projection properties of orthogonal arrays with strength three, Biometrika, 85, 491-495.

Das RN (1997). Robust second order rotatable designs: part-I, Calcutta Statistical Association Bulletin, 47, 199-214.

Das RN (2013). Discrepancy in classical lifetime model classes: Some illustrations, Journal of Quality, 20, 521-533.

Das RN and Huda S (2011). On D-optimal robust designs for exponential lifetime distribution, Journal of Statistical Theory and Applications, 10, 198-208. 
Das RN, Kim J, and Lee Y (2015). Robust first-order rotatable lifetime improvement experimental designs, Journal of Applied Statistics, 42, 1911-1930.

Das RN, Kim J, and Park JS (2015). Robust D-optimal designs under correlated error applicable invariantly for some lifetime distributions, Reliability Engineering \& System Safety, 136, 92100.

Das RN and Lee Y (2009). Log normal versus gamma models for analyzing data from qualityimprovement experiments, Quality Engineering, 21, 79-87.

Das RN and Lin DKJ (2011). On D-optimal robust first order designs for lifetime improvement experiments, Journal Statistical Planning and Inference, 141, 3753-3759.

Das RN and Park JS (2012). Discrepancy in regression estimates between log-normal and gamma: Some case studies, Journal of Applied Statistics, 39, 97-111.

Das RN, Park SH, and Aggarwal M (2010). On D-optimal robust second order slope-rotatable designs, Journal of Statistical Planning and Inference, 140, 1269-1279.

Firth D (1988). Multiplicative errors: log-normal or gamma?, Journal of the Royal Statistical Society, 50, 266-268.

Goos and Jones (2011). Optimal Designs of Experiments: A Case Study Approach, John Wiley and Sons, New York.

Lawless JF (1982). Statistical Models and Methods for Lifetime Data, John Wiley and Sons, New York.

Lee Y, Nelder JA, and Pawitan Y (2017). Generalized Linear Models with Random Effects (Unified Analysis via H-likelihood)(2nd ed.), Chapman \& Hall, London.

Myers RH and Carter WH (1973). Response surface techniques for dual response systems, Technometrics, 15, 301-317.

Myers RH, Montgomery DC, and Vining GG (2002). Generalized Linear Models with Applications in Engineering and the Sciences, John Wiley \& Sons, New York.

Nair VN et al. (1992). Taguchi's parameter design: A panel discussion, Technometrics, 34, 127-161.

Nelder JA and Lee Y (1991). Generalized linear models for the analysis of Taguchi-type experiments, Applied Stochastic Models and Data Analysis, 7, 107-120.

Watkins D, Bergman A, and Horton R (1994). Optimization of tool life on the shop floor using design of experiments, Quality Engineering, 6, 609-620. 
